\title{
Endoplasmic Reticulum Polymers Impair Luminal Protein Mobility and Sensitize to Cellular Stress in Alpha ${ }_{1}$-Antitrypsin Deficiency
}

\author{
Adriana Ordóñez, ${ }^{1}$ Erik L. Snapp, ${ }^{2}$ Lu Tan, ${ }^{1}$ Elena Miranda, ${ }^{1,3}$ Stefan J. Marciniak, ${ }^{1 *}$ and David A. Lomas ${ }^{1 *}$
}

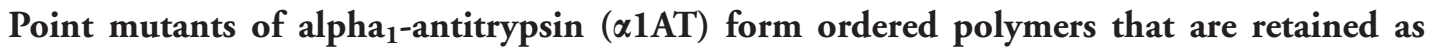
inclusions within the endoplasmic reticulum (ER) of hepatocytes in association with neonatal hepatitis, cirrhosis, and hepatocellular carcinoma. These inclusions cause cell damage and predispose to ER stress in the absence of the classical unfolded protein response (UPR). The pathophysiology underlying this ER stress was explored by generating cell models that conditionally express wild-type (WT) $\alpha 1 \mathrm{AT}$, two mutants that cause polymer-mediated inclusions and liver disease (E342K [the $\mathrm{Z}$ allele] and $\mathrm{H334D}$ ) and a truncated mutant (Null Hong Kong; NHK) that induces classical ER stress and is removed by ER-associated degradation. Expression of the polymeric mutants resulted in gross changes in the ER luminal environment that recapitulated the changes observed in liver sections from individuals with PI*ZZ $\propto 1$ AT deficiency. In contrast, expression of NHK $\alpha 1$ AT caused electron lucent dilatation and expansion of the ER throughout the cell. Photobleaching microscopy in live cells demonstrated a decrease in the mobility of soluble luminal proteins in cells that express $\mathrm{E} 342 \mathrm{~K}$ and $\mathrm{H334 \textrm {D }} \alpha 1 \mathrm{AT}$, when compared to those that express WT and NHK $\alpha 1 \mathrm{AT}(0.34 \pm 0.05,0.22 \pm 0.03,2.83 \pm 0.30$, and $2.84 \pm$ $0.55 \mu \mathrm{m}^{2} / \mathrm{s}$, respectively). There was no effect on protein mobility within ER membranes, indicating that cisternal connectivity was not disrupted. Polymer expression alone was insufficient to induce the UPR, but the resulting protein overload rendered cells hypersensitive to ER stress induced by either tunicamycin or glucose depletion. Conclusion: Changes in protein diffusion provide an explanation for the cellular consequences of ER protein overload in mutants that cause inclusion body formation and $\alpha 1 \mathrm{AT}$ deficiency. (Hepatology 2013;57:2049-2060)
\end{abstract}

A lpha ${ }_{1}$-antitrypsin $(\alpha 1 \mathrm{AT})$ deficiency results from point mutations that lead to the formation of large, ordered polymers that accumulate within the endoplasmic reticulum (ER) of hepatocytes. ${ }^{1}$ The resulting inclusions cause a toxic gain of function that is associated with neonatal hepatitis, cirrhosis, and hepato- cellular carcinoma. ${ }^{2}$ This is best exemplified by the $\mathrm{Z}$ (E342K), King's (H334D), Siiyama (S53F), and Mmalton $(\Delta 52 \mathrm{~F})$ alleles. $^{3-6}$ Plasma deficiency in $\alpha 1 \mathrm{AT}$ deficiency can also result from truncating mutations, such as the Null Hong Kong (NHK) allele that target the protein for ER-associated degradation (ERAD). ${ }^{7}$ This

Abbreviations: $\alpha 1 A T, \alpha_{1}$-antitrypsin; ATF6, activating transcription factor 6; CHO, Chinese hamster ovary; Deff effective diffusion coefficient; Dox, doxycycline; ELISA, enzyme-linked immunosorbent assay; EOR, endoplasmic overload response; ER, endoplasmic reticulum; ERAD, ER-associated degradation; FLIP, fluorescence loss in photobleaching; FRAP, fluorescence recovery after photobleaching; ICC, immunocytochemistry; IRE1, inositol-requiring 1; mRNA, messenger RNA; NHK, Null Hong Kong allele; PAGE, polyacrylamide gel electrophoresis; PERK, protein Kinase-like ER kinase; RFP, red fluorescent protein; RT-PCR, reverse-transcriptase polymerase chain reaction; SDS, sodium dodecyl sulfate; Tet-On, tetracycline-on; Tm, tunicamycin; UPR, unfolded protein response; XBP-1, X-box binding protein 1; WT, wild type.

From the ${ }^{1}$ Department of Medicine, University of Cambridge, Cambridge Institute for Medical Research, Cambridge, UK; ${ }^{2}$ Department Anatomy and Structural Biology, Albert Einstein College of Medicine of Yeshiva University, Bronx, NY; and 'Dipartimento di Biologia e Biotecnologie "Charles Darwin" e Istituto PasteurFondazione Cenci Bolognetti, Università di Roma "La Sapienza," Rome, Italy.

Received August 3, 2012; revised October 23, 2012; accepted November 9, 2012.

This work was supported by the postdoctoral fellowship grant from the eALTA Foundation (Griffols; to A.O.). S.J.M. is a Medical Research Council (MRC) Senior Clinical Fellow (G0601840). E.L.S. is supported by the National Institute of General Medical Sciences (R01GM086530-01). D.A.L. is supported by the MRC (UK), the Cambridge National Institute for Health Research (NIHR) Biomedical Research Center, the Engineering and Physical Sciences Research Council (UK), and Papworth National Health Service Trust. L.T. is supported by the NIHR Biomedical Research Center and the British Research Council. E.M. is supported by the Istituto Pasteur-Fondazione Cenci Bolognetti and by the Telethon Foundation, Italy.

*Joint senior authors. 
reduces secretion of $\alpha 1 \mathrm{AT}$, but does not cause the gainof-function liver disease. In both cases, the lack of circulating $\alpha 1$ AT predisposes the homozygote to early-onset emphysema. ${ }^{8}$

A wide range of signaling pathways are activated to restore the ER homeostasis in response to an overload of misfolded proteins. An important stress response is the unfolded protein response (UPR) that combines transient attenuation of protein translation with enhancement of the ER's protein-folding capacity, thus preventing uncontrolled aggregation of proteins within its lumen.' This conserved stress response is tightly regulated by the activation of three ER stress sensors operating in parallel: inositol-requiring 1 (IRE1); double-stranded RNA-activated protein kinase-like ER kinase (PERK); and activating transcription factor 6 (ATF6). Under nonstressed conditions, each sensor is held in an inactive state by binding of the ER chaperone, BiP, to its luminal domain. When misfolded protein levels increase during ER stress, the UPR is activated, at least in part, through the titration of $\mathrm{BiP}$ away from these sensor molecules.

We aimed to determine the effect of polymers and truncated $\alpha 1 \mathrm{AT}$ on the ER environment. We show that the accumulation of ordered polymers of $\alpha 1 \mathrm{AT}$ leads to gross expansion of ER cisternae and impairment of ER luminal mobility. This hinders the organelle's capacity to deal with otherwise minor perturbations of protein folding and thus leads to enhanced sensitivity to ER stress. Our findings provide one of several potential mechanisms for the toxicity associated with ER overload in $\alpha 1$ AT deficiency-related liver disease.

\section{Materials and Methods}

Plasmid Constructs. Complementary DNA for human wild-type (WT) $\alpha 1 \mathrm{AT}$ and two polymeric mutants (E342K and $\mathrm{H} 334 \mathrm{D})$ were subcloned into the pTRE2hyg vector (Clontech, Saint-Germain-enLaye, France) for the generation of stable Chinese hamster ovary (CHO)-K1 Tet-On (tetracycline-on) $\alpha 1$ AT cell lines. The NHK-truncated $\alpha 1$ AT variant (L318fsX17) was generated with the QuickChange Site-Directed Mutagenesis kit (Stratagene, La Jolla, CA) using WT $\alpha 1 \mathrm{AT}$ as the template and then subcloned into the pTRE2hyg plasmid.
Generation and Characterization of Stable Cell Lines Expressing a1AT. The CHO-K1 cell line was purchased from Clontech, and stable cell lines were generated by following the manufacturer's instructions. Cells were maintained in Dulbecco's modified Eagle's medium supplemented with $10 \%(\mathrm{v} / \mathrm{v})$ Tet/fetal bovine serum, $1 \%(\mathrm{w} / \mathrm{v})$ nonessential amino acids, $1 \%(\mathrm{w} / \mathrm{v})$ penicillin/streptomycin, $200 \mu \mathrm{g} / \mathrm{mL}$ of Geneticin, and $500 \mu \mathrm{g} / \mathrm{mL}$ of Hygromycin B (both selective antibiotics from Invitrogen, Carlsbad, CA) at $37^{\circ} \mathrm{C}$ and $5 \%$ (v/v) $\mathrm{CO}_{2} . \alpha 1 \mathrm{AT}$ was typically induced with $1 \mu \mathrm{g} / \mathrm{mL}$ of doxycycline (Dox) for 48 hours. Whole cell lysates, sodium dodecyl sulfate (SDS) and nondenaturing polyacrylamide gel electrophoresis (PAGE) followed by immunoblotting, enzyme-linked immunosorbent assay (ELISA), and metabolic labeling were performed as detailed previously ${ }^{4,10}$ (see Supporting Materials).

Immunofluorescence, Electron Microscopy, and Immunocytochemistry. Immuno- and electron microscopy of CHO-K1 cells and immunocytochemistry (ICC) of paraffin tissue slides from human subjects were undertaken as described in the Supporting Materials.

Photobleaching Analysis in Live Cells. CHO-K1 Tet-On cells were transiently transfected with an ERRFP (red fluorescent protein) plasmid ${ }^{11}$ or a CytERM (cytoplasmic end of an ER signal-anchor membrane protein) msfGFP (monomeric superfolder green fluorescent protein) membrane reporter. ${ }^{12}$ Live cells were imaged on a $37^{\circ} \mathrm{C}$ environmental controlled stage of a confocal microscope system (DuoScan; Carl Zeiss Inc., Thornwood, NY) with a $\times 63 / 1.4 \mathrm{NA}$ oil objective and a 40-mW 561-nm diode laser with a 565-nm bandpass filter. For fluorescence recovery after photobleaching/fluorescence loss in photobleaching (FRAP/FLIP) experiments, a small region of interest was photobleached at full laser power in the 561 line and fluorescence recovery or loss was monitored over 0.2 -second time intervals. Effective diffusion coefficient $\left(D_{\text {eff }}\right)$ measurements were calculated as described previously. ${ }^{13}$ Fluorescence loss curves were obtained by transforming fluorescence intensities into a percentage scale in which the first bleach time point represents $100 \%$ of fluorescence intensity. Statistical significance was assessed using the Student $t$ test.

Address reprint requests to: Stefan J. Marciniak, Department of Medicine, University of Cambridge, Cambridge Institute for Medical Research, Wellcome Trustl MRC Building, Hills Road, Cambridge, CB2 OXY, UK. E-mail: sjm20@cam.ac.uk; fax: +44 (0)1223 336827.

Copyright $(2012$ by the American Association for the Study of Liver Diseases.

View this article online at wileyonlinelibrary.com.

DOI 10.1002/hep.26173

Potential conflict of interest: Nothing to report.

Additional Supporting Information may be found in the online version of this article. 
Detection of the UPR Markers. Immunoblottings for three main ER luminal chaperone proteins (GRP94, BiP/GRP78, and PDI), the ATF6-reporter luciferase assay, and the reverse-transcriptase polymerase chain reaction (RT-PCR) X-box binding protein 1 (XBP-1) splicing assay were performed as described previously. ${ }^{10}$

\section{Results}

Conditional Expression of $\alpha \mathbf{1 A T}$. Stable CHO-K1 cell lines were developed using the Tet-On inducible system that enables tight regulation of $\alpha 1$ AT expression. Four clonal cell lines were generated to express four single variants of $\alpha 1 \mathrm{AT}$ : the WT human $\alpha 1 \mathrm{AT}$ $(\mathrm{M})$; the polymeric $\mathrm{Z}$ [E342K] mutant; the polymeric King's [H334D] mutant (both form-ordered polymers in the ER of hepatocytes in association with liver disease $\left.^{3,4}\right)$; and an NHK-truncated variant that does not fold properly, preventing the formation of ordered polymers, and instead activates the UPR. ${ }^{7}$ Expression of each variant was readily detected on treating cells with Dox (Figs. 1 and Supporting Fig. 1A-E). Cells expressing $M \alpha 1 \mathrm{AT}$ showed lower intracellular signal, compared to medium, consistent with its efficient secretion. Nondenaturing PAGE and ELISA assays identified intracellular polymers for only $Z$ and $\mathrm{H} 334 \mathrm{D} \alpha 1 \mathrm{AT}$. No ELISA signal was detected for the NHK variant, suggesting that the 9C5 epitope that is present in mono- and polymeric forms of $\alpha 1 \mathrm{AT}$ was removed by deletion of the C-terminal 61 amino acids. Protein-trafficking rate was assayed by metabolic labelling, followed by immunoprecipitation (Fig. 1C). WT $\alpha 1$ AT was almost completely secreted, compared with $\mathrm{Z}$ and $\mathrm{H} 334 \mathrm{D} \alpha 1 \mathrm{AT}$, which were largely retained as polymers. In contrast, NHK $\alpha 1 \mathrm{AT}$ failed to reach the culture medium, despite being cleared from cell lysates at 4 hours, consistent with its degradation by ERAD. $^{7}$

Accumulation of a1AT Polymers Causes Disruption of the ER. Immunofluorescence confocal microscopy revealed that both $\mathrm{Z}$ and H334D $\alpha 1 \mathrm{AT}$ colocalized with the ER marker, BiP, although, in many cells, ER structure was distorted from its reticular morphology into a punctate pattern (Fig. 2A). No gross morphological changes were observed in cells expressing either $\mathrm{M}$ or NHK $\alpha 1 \mathrm{AT}$. Of note, only $\mathrm{M}$ $\alpha 1$ AT colocalized with the Golgi marker, p115, indicating efficient exit from the ER (Fig. 2A). Electron microscopy was used to visualize cells expressing WT and mutant $\alpha 1 \mathrm{AT}$. The ultrastructure in cells expressing $\mathrm{M} \alpha 1 \mathrm{AT}$ was normal (Fig. 2B,a-b), when they were compared with mock-transfected cells (Fig. 2B,i$\mathrm{k})$. The inclusions in cells expressing either $\mathrm{Z}$ or $\mathrm{H} 334 \mathrm{D} \propto 1 \mathrm{AT}$ were associated with the replacement of healthy tubular and lamellar ER by electron-dense vesiculated structures (Fig. 2B,c-f). In contrast, expression of the NHK variant induced a markedly different phenotype with electron lucent dilatation and expansion of the ER throughout the cell (Fig. 2B,g-h). To elucidate whether the alterations observed could be the result of a massive overexpression of $\alpha 1 \mathrm{AT}$ in our model, we also assessed the distribution and signal of total $\alpha 1 \mathrm{AT}$ in paraffin-embedded liver sections from a PI*ZZ $\alpha 1$ AT homozygote and control human liver by ICC (Fig. 2C). The PI*ZZ homozygote human liver demonstrated levels of polymer accumulation at least as dramatic as that noted in cultured cells, suggesting that the CHO-K1 Tet-On cells represent a valid model of the accumulation of $\alpha 1$ AT polymers within the ER. Taken together, these data show that the accumulation of ordered polymers within the ER leads to gross changes in the organelle's morphology and, importantly, that these changes differ markedly from those induced by the NHK mutant that causes classical ER stress.

Polymeric Mutants of a1AT Impair ER Protein Mobility in Live Cells. The effect of polymer formation on the ER luminal environment was determined by monitoring changes in the mobility of the ER-RFP fluorescence marker in live cells. ER-localized RFP is a protein $(27-\mathrm{kDa}$ and $2.3-\mathrm{nm}$ hydrodynamic radius) with no known interacting partners that can rapidly sample the entire ER lumen, reporting on both crowdedness and interconnectivity. ${ }^{11}$ ER-RFP distribution revealed two distinct ER architectures in cells expressing $\alpha 1 \mathrm{AT}$ variants. Each cell line displayed a typical ER reticular network in the absence of Dox, as did cells expressing $\mathrm{M}$ and NHK $\alpha 1 \mathrm{AT}$ when treated with Dox (Supporting Fig. 2). In contrast, approximately $30 \%-40 \%$ of cells expressing $\mathrm{Z}$ and H334D $\alpha 1 \mathrm{AT}$ developed a characteristic vesiculated architecture when treated with Dox (Supporting Fig. 2), as detected by staining for either $\alpha 1 \mathrm{AT}$ or BiP (Fig. 2A). Within the same clonal cell lines, there remained a proportion of cells expressing $\mathrm{Z}$ and $\mathrm{H} 334 \mathrm{D} \alpha 1 \mathrm{AT}$ that retained the normal reticular pattern of ER, suggesting that formation of inclusion bodies is a stochastic phenomenon (Supporting Fig. 3).

We then assessed ER lumen continuity by FLIP, ${ }^{14}$ in which a small region of cell ER-RFP fluorescence was repeatedly photobleached. If the protein is mobile within a continuous compartment, total fluorescence within this compartment will be depleted. Over time, 


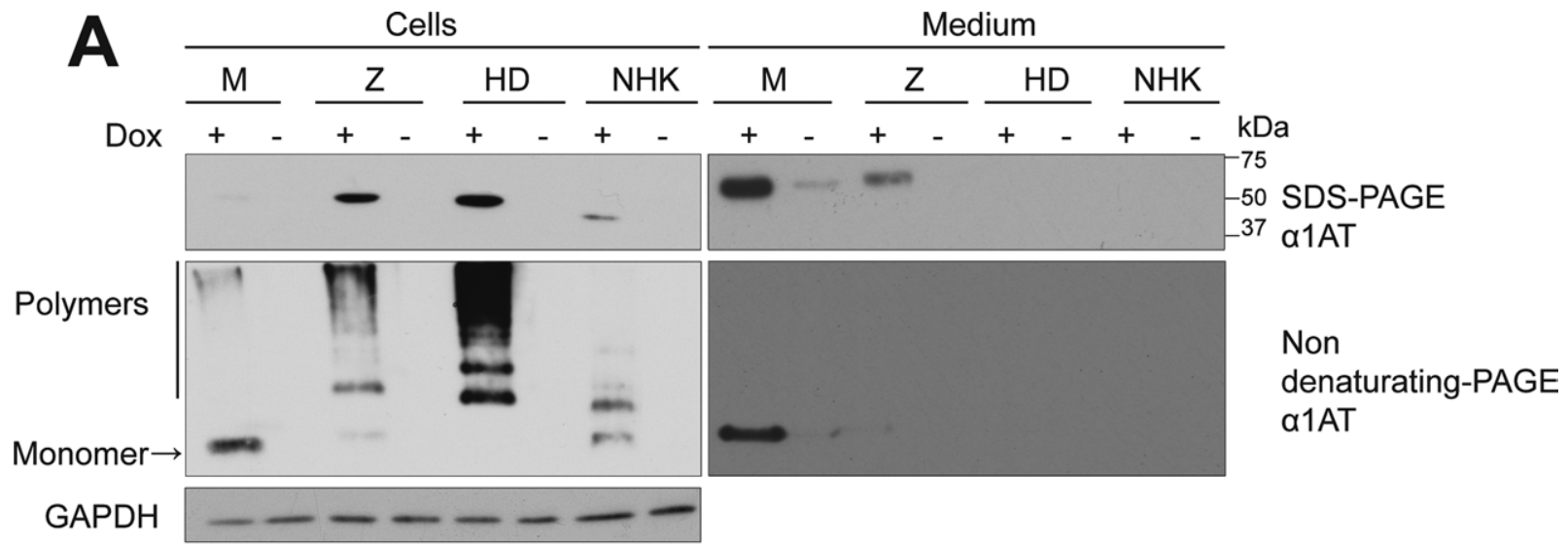

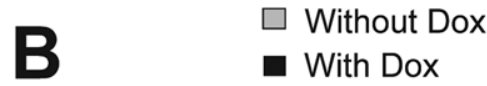

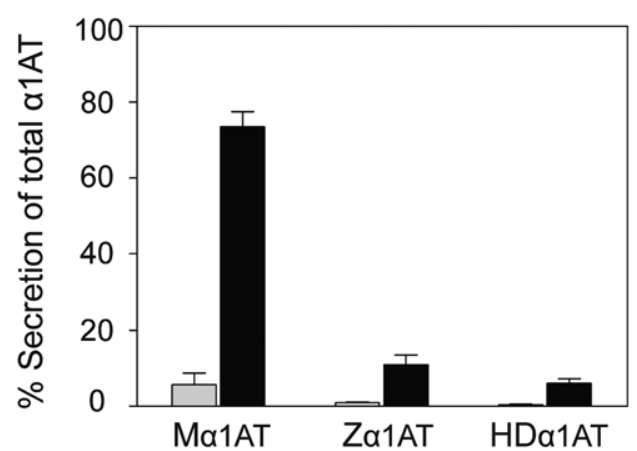

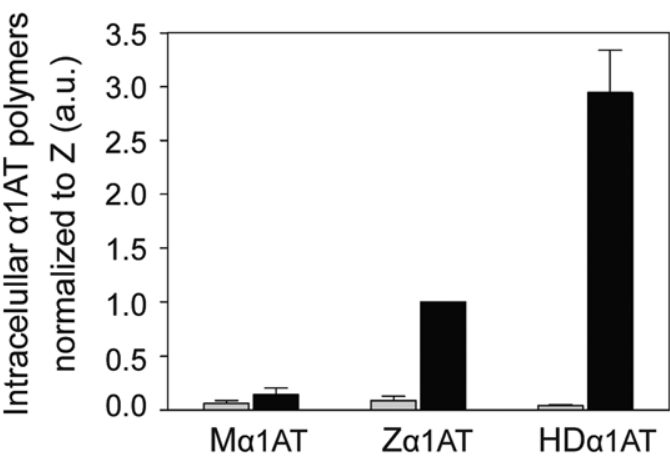

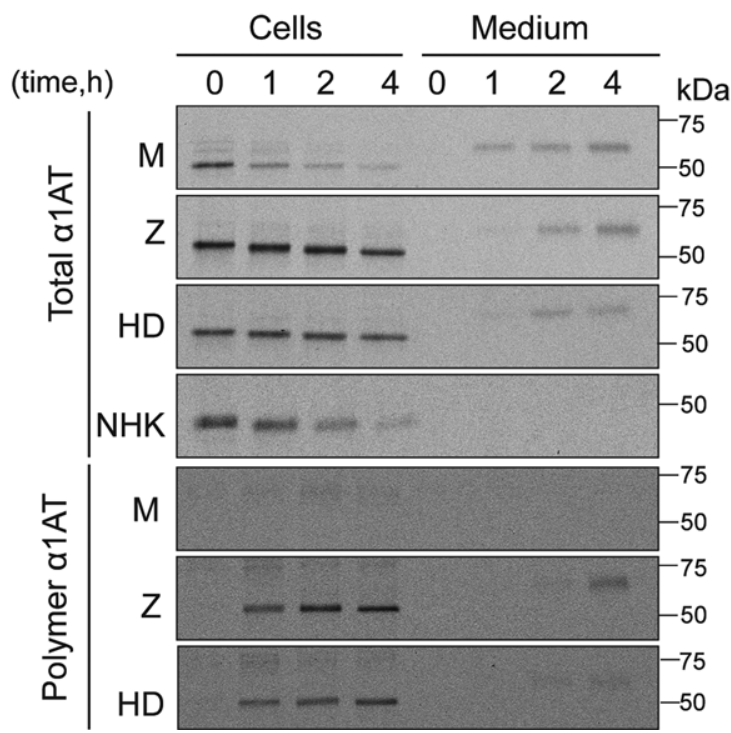

Fig. 1. Characterization of CHO-K1 Tet-On cell lines expressing different variants of $\alpha 1 \mathrm{AT}$ : WT (M); Z (E342K); HD (H334D); and the truncated NHK variant. (A) Cell lysates and medium were collected after induction of $\alpha 1 \mathrm{AT}$ with $1 \mu \mathrm{g} / \mathrm{mL}$ of doxycycline (Dox) for 48 hours and analyzed by $10 \%$ (w/v) SDS and nondenaturing PAGE, followed by immunoblotting for $\alpha 1$ AT. (B) Percentage of secreted total $\alpha 1$ AT and quantification of intracellular polymers for $\mathrm{M}, \mathrm{Z}$, and HD $\alpha 1 \mathrm{AT}$ were quantified by sandwich ELISA using the 9C5 (left panel) or 2C1 (right panel) monoclonal antibodies, which recognize all forms of $\alpha 1 \mathrm{AT}$ or only polymers, respectively. Graphs show mean \pm standard error of the mean $(\mathrm{n}=4)$. (C) CHO-K1 Tet-On cells expressing M, Z, HD, and NHK $\alpha 1$ TT were pulse-labeled with ${ }^{35} \mathrm{~S}$-Met/Cys for 15 minutes and chased for the times indicated (0-4 hours). $\alpha 1 \mathrm{AT}$ from cell lysates and medium was immunoprecipitated with a polyclonal antibody (total) or the $2 \mathrm{C} 1$ monoclonal antibody (polymers), then samples were resolved by $10 \%$ (w/v) SDS-PAGE and detected by autoradiography. Gels are representative of three independent experiments. 

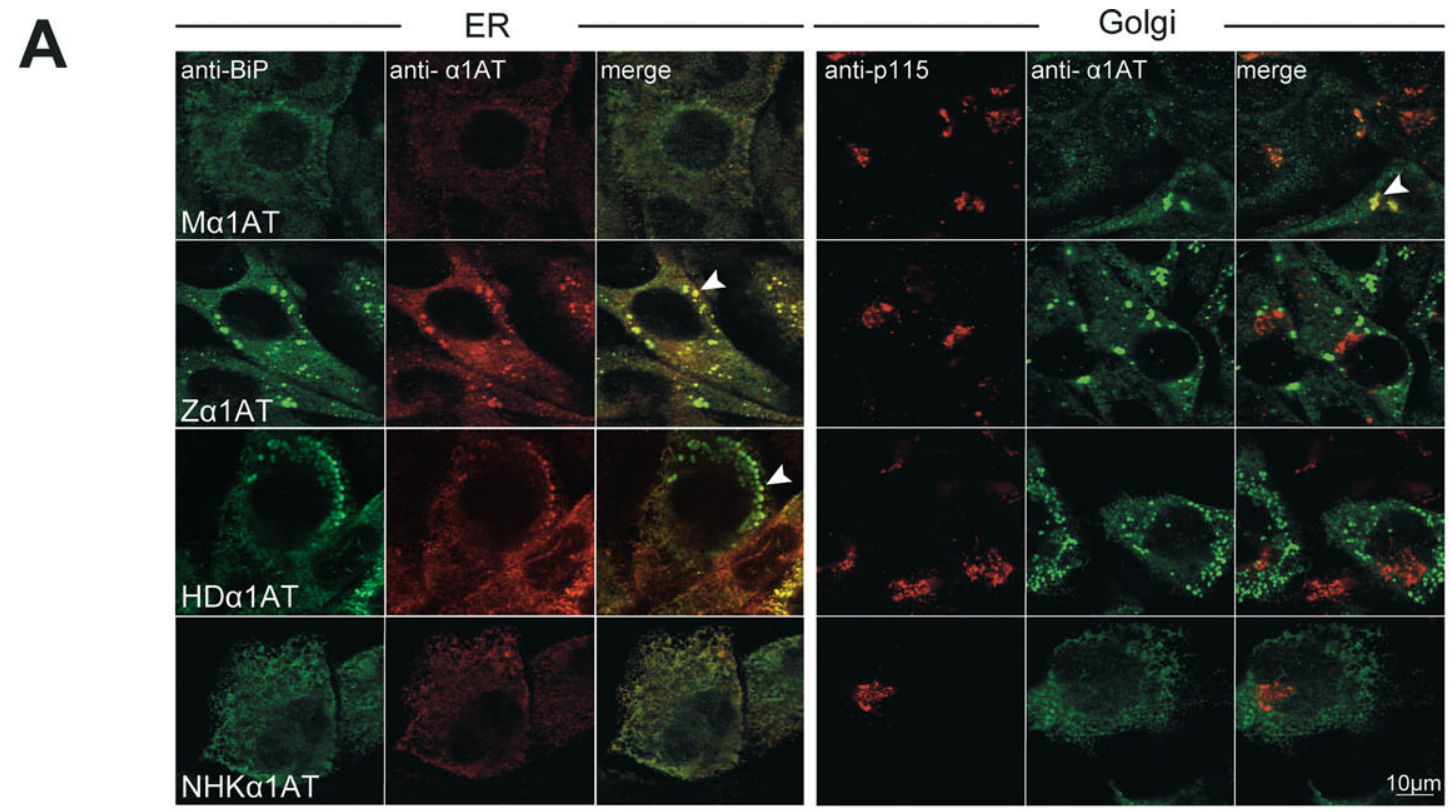

B
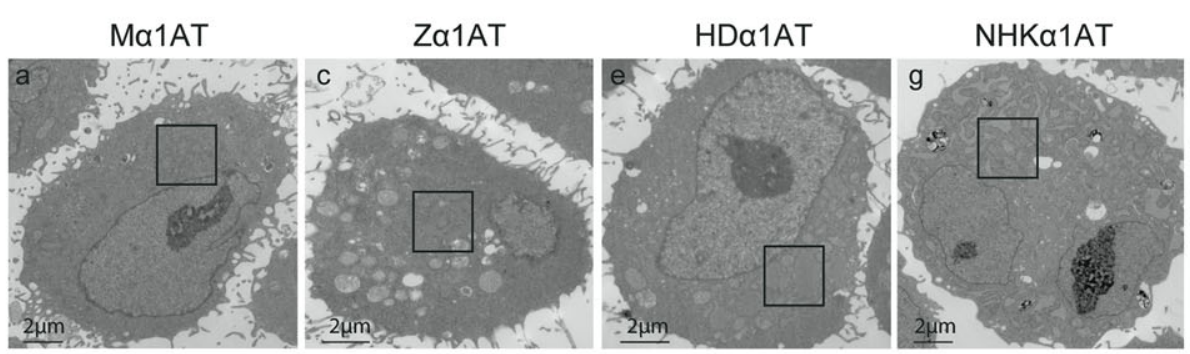

Mock-transfected
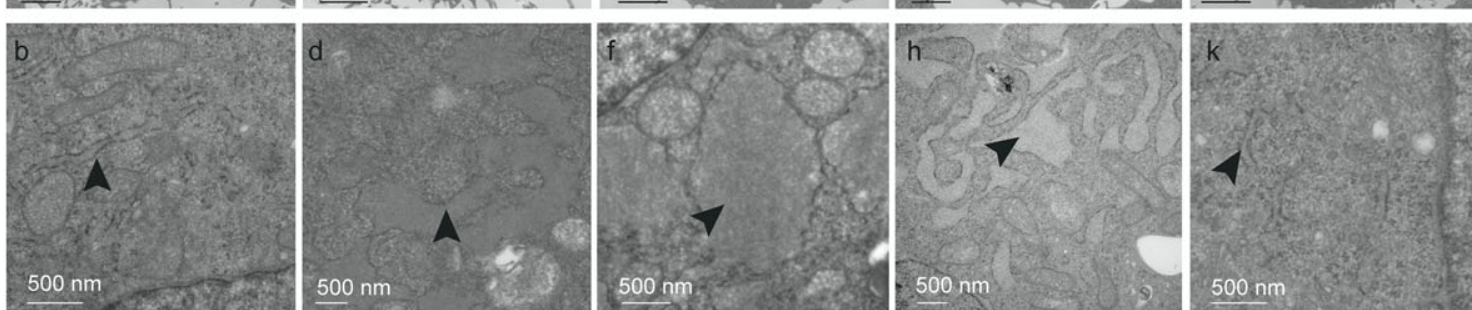

C
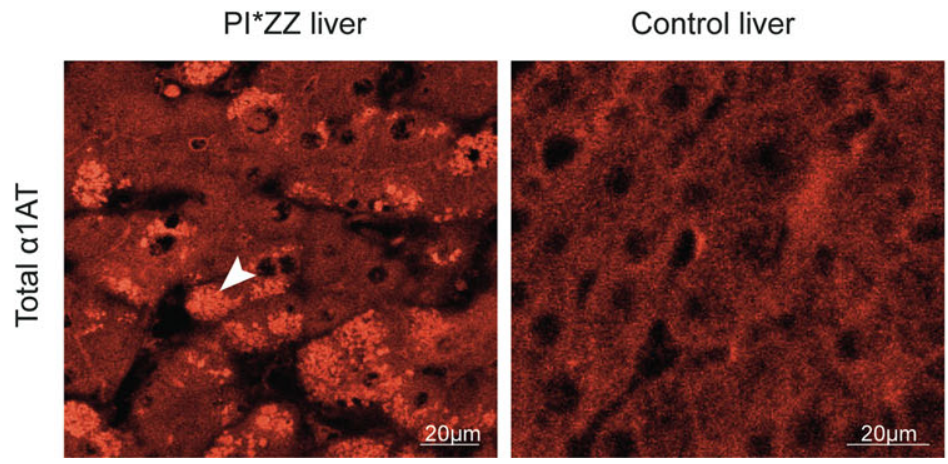

Fig. 2. Intracellular accumulation of $\alpha 1 A T$ polymers in inclusion bodies disrupts ER structure. (A) Representative fluorescence images of TetOn CHO-K1 cells were induced to express M, Z, HD, and NHK $\alpha 1$ AT with Dox for 48 hours, fixed, and coimmunostained with an $\alpha 1 A T$ polyclonal antibody and either an ER protein marker (BiP) or a Golgi protein marker (p115). White arrows in merged panels show a punctate pattern of colocalized polymerogenic forms of $\alpha 1 A T$ ( $Z$ and $H D$ ) with the ER marker (left panel) or WT $\alpha 1 A T$ colocalized with the Golgi marker (right panel). (B) Electron microscopy analysis for ER structure in Tet-On CHO-K1 cells expressing $\alpha 1 \mathrm{AT}$ variants. Upper panels represent whole cells, with higher power images shown in lower panels. Black arrows indicate ER cisterns. Inclusion bodies were only identified in micrographs of polymerogenic mutants ( $Z$ and $\mathrm{HD} \alpha 1 \mathrm{AT}$ ). (C) Immunostaining of paraffin-embedded liver sections from a $\mathrm{PI} Z \mathrm{ZZ} \alpha 1 \mathrm{AT}$ individual and a healthy control liver with a polyclonal antibody to total $\alpha 1 \mathrm{AT}$ (white arrow). 
ER-RFP fluorescence in the whole cell was steadily and uniformly lost in cells displaying a tubular ER network, whereas fluorescence depletion was substantially slower in cells containing $\mathrm{Z} \alpha 1 \mathrm{AT}$ polymer (Fig. 3A; Supporting Videos 1 and 2). This did not reflect an effect of transfected ER-RFP expression levels, because normalized plots showed that the remaining whole cell fluorescence intensity after 200 seconds was $23.5 \%$ and $61.1 \%$ for cells retaining a tubular ER network or a polymer-filled ER, respectively (Fig. 3A). Similar results were obtained for cells expressing H334D $\alpha 1$ AT (Fig. 3B). Thus, the presence of vesiculated structures is associated with marked loss of intraER luminal communication (i.e., slower ER-RFP mobility between inclusions) that is likely to adversely increase the heterogeneity of the ER luminal environment.

FRAP analysis was used to quantify the change in mobility of the ER-RFP reporter (Fig. 4A). This provides information on local rates of protein diffusion $\left(D_{\text {eff }}, \mu \mathrm{m}^{2} / \mathrm{s}\right)$ by bleaching a small defined region of a single cell. $D_{\text {eff }}$ is affected by local viscosity, size of the reporter protein, and its protein-protein interactions ${ }^{15}$ and is inversely proportional to crowdedness of the environment. Moreover, small changes in $D_{\text {eff }}$ are likely to be biologically significant. ${ }^{11}$ No significant differences in mobility of ER-RFP were detected in the different cell lines in the absence of $\alpha 1$ AT expression (Fig. $4 \mathrm{~B})$. ER-RFP $D_{\text {eff }}$ values in cells expressing NHK and $\mathrm{M} \propto 1 \mathrm{AT}$ were identical $(2.84 \pm 0.30$ versus $2.83 \pm$ $0.55 \mu \mathrm{m}^{2} / \mathrm{s}$ ). However, cells containing polymers of either $\mathrm{Z}$ or $\mathrm{H} 334 \mathrm{D}$ a1AT exhibited significantly lower ER-RFP $D_{\text {eff }}$ values, compared with $\mathrm{M} \alpha 1 \mathrm{AT}(0.34 \pm$ 0.05 and $0.22 \pm 0.03 \mu \mathrm{m}^{2} / \mathrm{s}$, respectively; Fig. 4A,B; Supporting Videos 2 and 3).

The striking effect of polymers on ER-RFP diffusion within the lumen of the ER prompted an assessment of their effect on diffusion of an ER transmembrane reporter. We used the CytERM msfGFP plasmid, which encodes a signal domain required to anchor the fluorescent protein into the ER membrane with the sfGFP exposed to the cytoplasm. ${ }^{12}$ There is no luminal domain and, consequently, the luminal content has no effect on membrane protein diffusion. There were no significant differences in CytERM msfGFP $D_{\text {eff }}$ values in cells containing polymers of $\mathrm{H} 334 \mathrm{D} \propto 1 \mathrm{AT}$, compared to cells in which expression was not induced $(0.35 \pm 0.04$ versus $0.40 \pm 0.02$ $\mu \mathrm{m}^{2} / \mathrm{s}$ ) (Fig. 4C).

Taken together, these data demonstrate that inclusions of $\alpha 1 \mathrm{AT}$ polymers have a remarkable effect on the ER environment, reducing luminal protein diffu- sion. However, protein mobility within ER membranes is unaffected, indicating that cisternae connectivity is not disrupted.

Increased Susceptibility to ER Stress in PolymerExpressing Cells. Previous work has shown that the ER accumulation of ordered polymers of mutant $\alpha 1 \mathrm{AT}$ and another member of the same protein family, neuroserpin, fail to activate the UPR. ${ }^{16-19}$ However, a recent report has suggested that $\mathrm{Z} \propto 1 \mathrm{AT}$ polymer accumulation triggers UPR activation in peripheral blood monocytes. ${ }^{20}$ We hypothesized that the alteration of the ER luminal environment might destabilize ER homeostasis sufficiently to sensitize the cell to a second insult and so to trigger activation of the UPR. Activation of multiple reporters of UPR signaling was monitored after treatment with tunicamycin (Tm), an inhibitor of N-linked glycosylation. Immunoblotting analysis for three ER luminal chaperones (BiP/GRP78, GRP94, and PDI) confirmed that ordered polymers of $\alpha 1 \mathrm{AT}$ alone failed to activate the UPR (Fig. 5A, lanes $1-3)$. In contrast and as expected, synthesis of truncated NHK $\alpha 1 A T$ led to robust activation of the UPR (Fig. 5A, lane 4). Remarkably, both polymerogenic a1AT variants showed a hypersensitive response in UPR activation after treatment with low concentrations of $\mathrm{Tm}(5-25 \mathrm{ng} / \mathrm{mL})$, when compared to the WT protein (Fig. 5A).

A more-quantitative reporter assay was used to assess the activation of the ATF6 branch of the UPR. Only cells expressing NHK $\alpha 1$ AT showed measureable activation of ATF6 under nonstressed conditions (Fig. 5B). However, cells expressing polymeric variants were more susceptible to activation of ATF6 on treatment with moderate concentrations of $\mathrm{Tm}(60-300 \mathrm{ng} / \mathrm{mL})$. A similar response was observed when cells were treated with low concentrations of glucose (10.0-0.2 $\mathrm{mM}$ ), which represents a more-physiological ER stress (Fig. 5C).

IRE1 activation was assessed by the splicing of $X B P 1$ messenger RNA (mRNA). None of the cell lines showed spliced XBP1 mRNA under basal conditions. However, Tm $(300 \mathrm{ng} / \mathrm{mL})$ efficiently triggered IRE1 in all cell lines, as reported by the appearance of a smaller spliced XBP1 mRNA band (Fig. 5D). The failure to detect splicing of XBP1 mRNA in cells induced to express the NHK variant in the absence of $\mathrm{Tm}$ reflects the transitory nature of IRE1 activation. ${ }^{21}$ After the induction of ER chaperones, which occurs rapidly on expressing this truncated mutant, ER stress is alleviated and IRE1 returns to its inactive state. Indeed, the elevated level of ER chaperones in NHKexpressing cells served to protect them from 


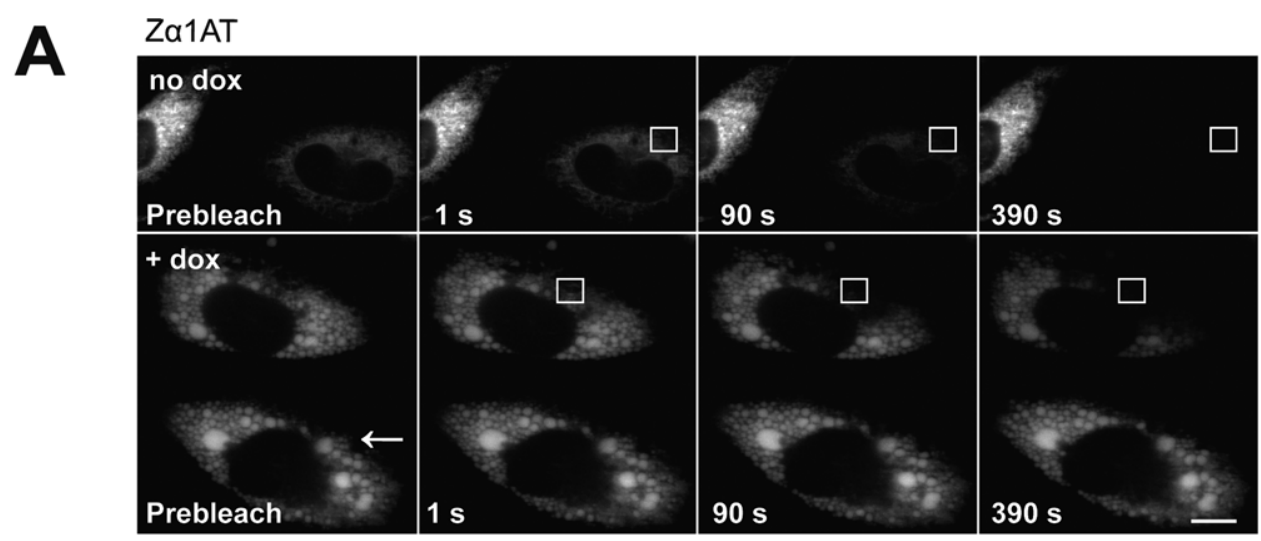

\section{ER-RFP}
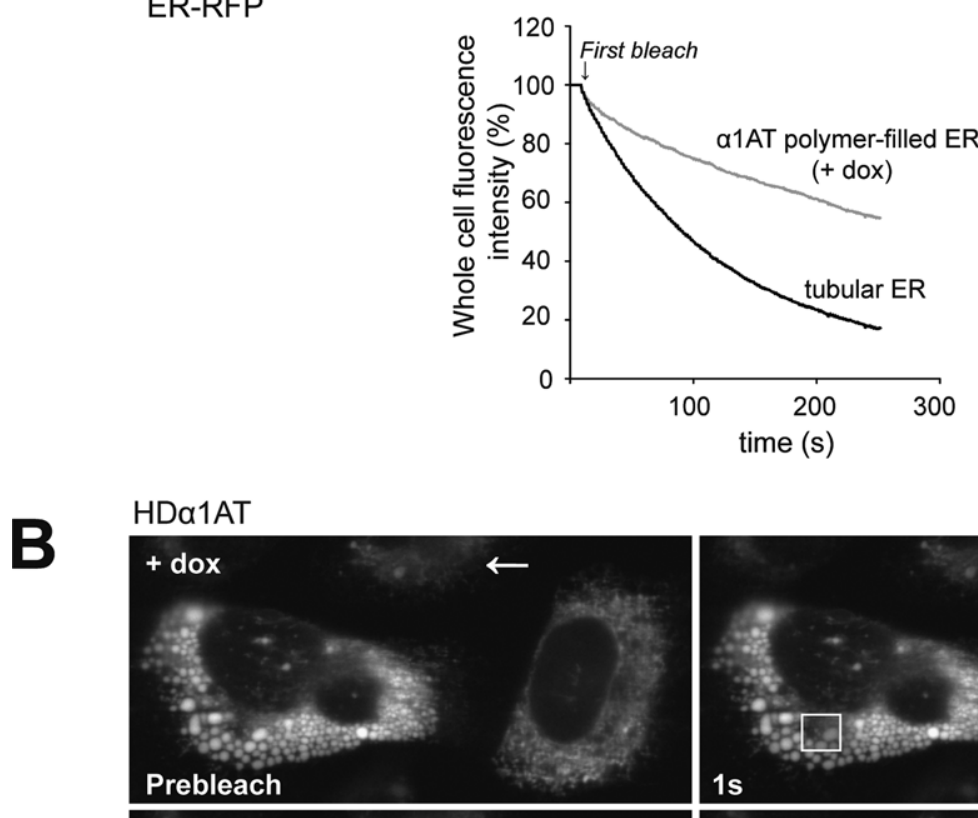

Fig. 3. Luminal $\alpha 1 A T$ inclusion bodies impair protein mobility within the ER lumen. (A) FLIP analysis of $Z$ $\alpha 1$ AT cells expressing ER-RFP within both a tubular ER network cell (without Dox) and an $\alpha 1 \mathrm{AT}$ polymer-filled ER (with Dox for 48 hours). FLIP normalized plots setting the prebleach intensity as $100 \%$. (B) FLIP of H334D $\alpha 1 \mathrm{AT}$ cells expressing ER-RFP within both a tubular ER network cell and an $\alpha 1 \mathrm{AT}$ polymer-filled ER from the same sample after treatment with dox for 48 hours. Normalized plots from FLIP analysis as described in (A). Fluorescence loss was specific, because adjacent cells remained fluorescent (white arrow). White boxes represent the region subjected to photobleaching.

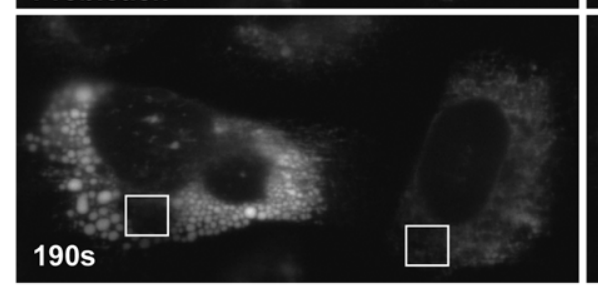

$1 \mathrm{~s}$

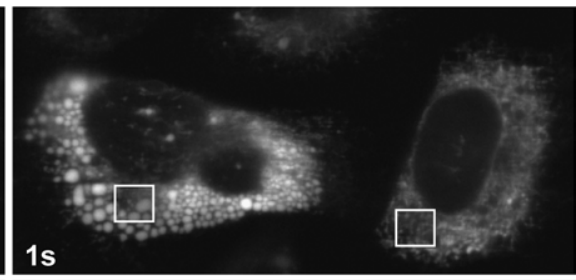

ER-RFP

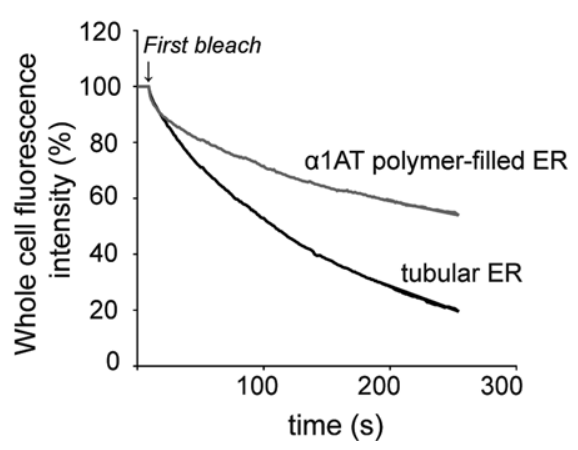

mRNA. These data indicate that the presence of polymers does not affect the intrinsic activity of IRE1. However, they do impair the cell's ability to restore ER homeostasis, because higher levels of spliced XBP1

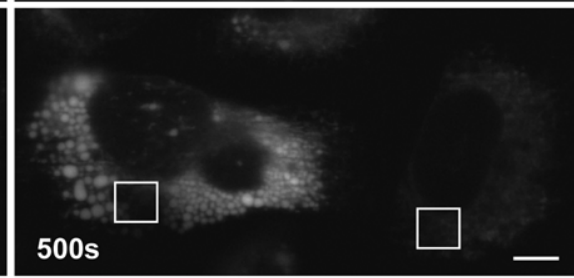

500 s subsequent ER stress, as evidenced by the blunted response of XBP1 splicing noted in these cells (Fig. 5D). In contrast, on treatment with Tm, M-, Z-, and H334D-expressing cells showed rapid splicing of XBP1 


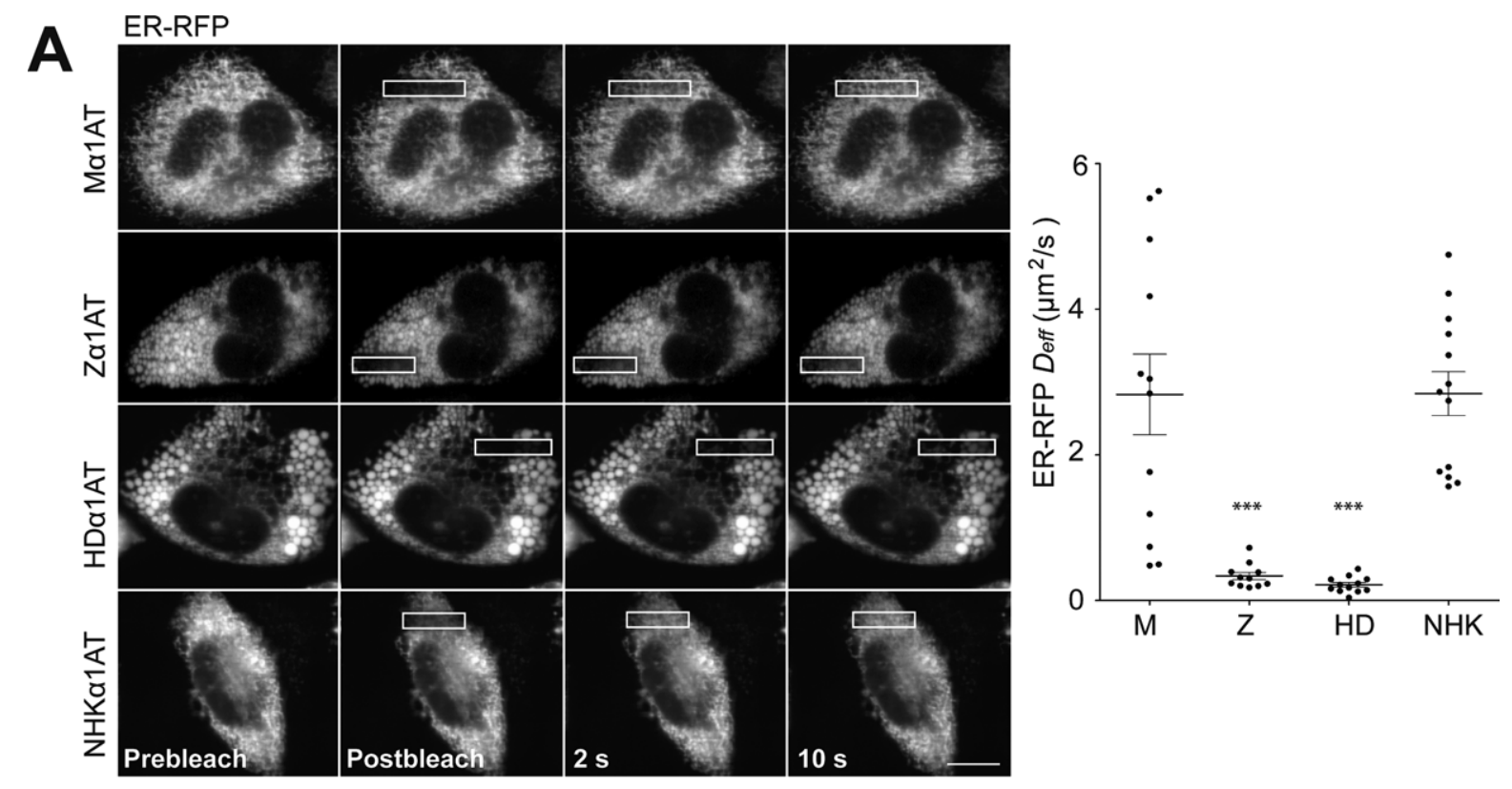

B

\begin{tabular}{|c|c|c|c|}
\hline Condition & Variant & No & ER-RFP Deff $\left(\mu \mathrm{m}^{2} / \mathrm{s}\right) \pm$ S.E.M \\
\hline \multirow{4}{*}{ With dox } & Ma1AT & 12 & $2.83 \pm 0.55$ \\
\hline & Za1AT & 11 & $0.34 \pm 0.05^{\star \star \star}$ \\
\hline & HDa1AT & 12 & $0.22 \pm 0.03^{\star \star \star *}$ \\
\hline & NHKa1AT & 12 & $2.84 \pm 0.30$ \\
\hline \multirow[t]{4}{*}{ Without dox } & Ma1AT & 12 & $3.04 \pm 0.29$ \\
\hline & Za1AT & 10 & $3.60 \pm 0.45$ \\
\hline & HDa1AT & 9 & $3.18 \pm 0.70$ \\
\hline & NHKa1AT & 11 & $2.03 \pm 0.30$ \\
\hline
\end{tabular}

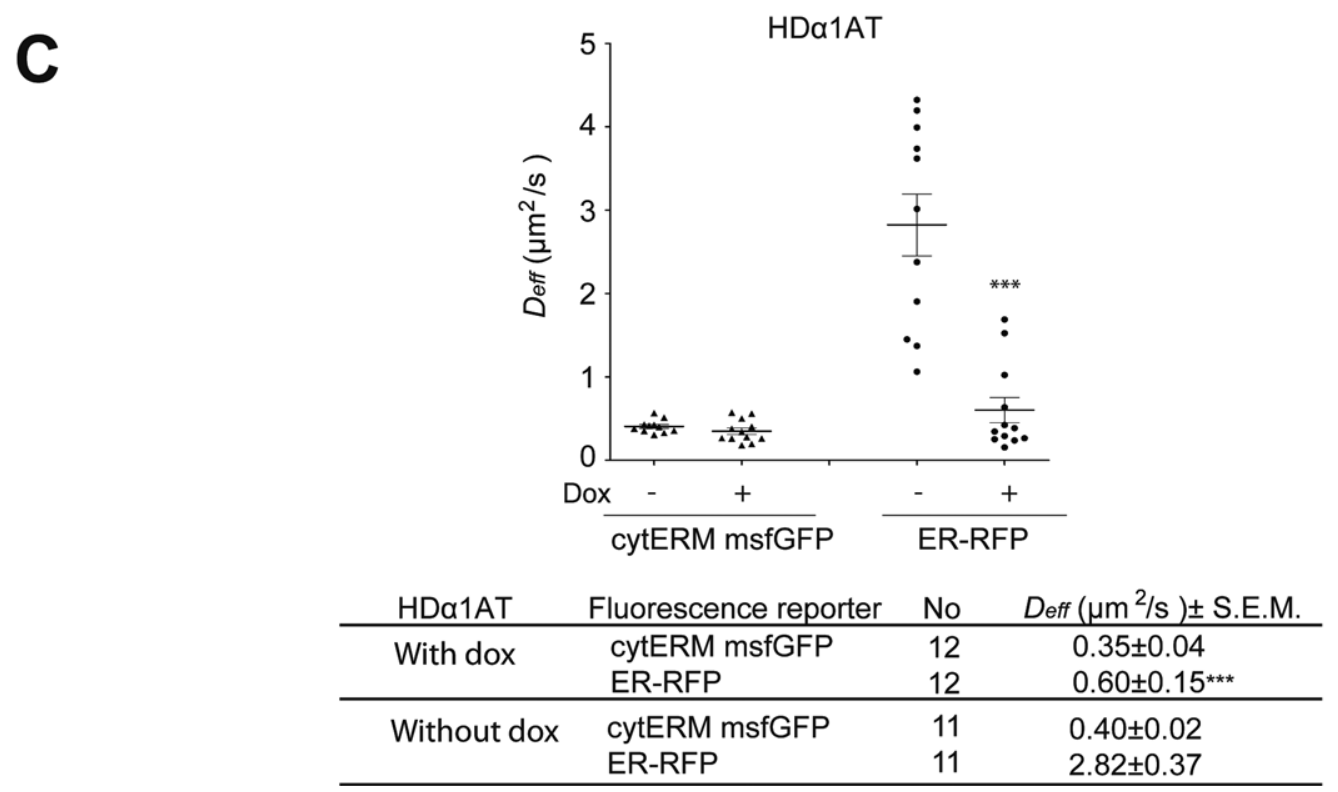

Fig. 4. Polymers of $\alpha 1 A T$ modify the ER luminal environment decreasing ER protein mobility. (A) Left: FRAP series of Tet-On CHO-K1 cells induced to express M, Z, HD, and NHK $\alpha 1 A T$ with Dox for 48 hours and transiently cotransfected with ER-RFP. Cells expressing either Z or HD $\alpha 1 A T$ show a vesiculated pattern in which fluorescence recovery was significantly reduced. All images were captured immediately before (prebleach), immediately after (postbleach), and at times after photobleaching in the white box. Scale bar: $10 \mu \mathrm{m}$. Right: $D_{\text {eff }}$ values from FRAP analysis in individual cells. (B) Mean values \pm standard error of the mean (SEM) for each condition and variant in (A). (C) Upper panel: $D_{\text {eff }}$ values from FRAP analysis in individual Tet-On HD CHO-K1 cells treated with or without Dox for 48 hours and transiently cotransfected with both ER-RFP and CytERM msfGFP membrane reporter. Lower panel: mean \pm SEM for each condition and construct. $* * * P<0.0001$ in a two-tailed Student $t$ test. 


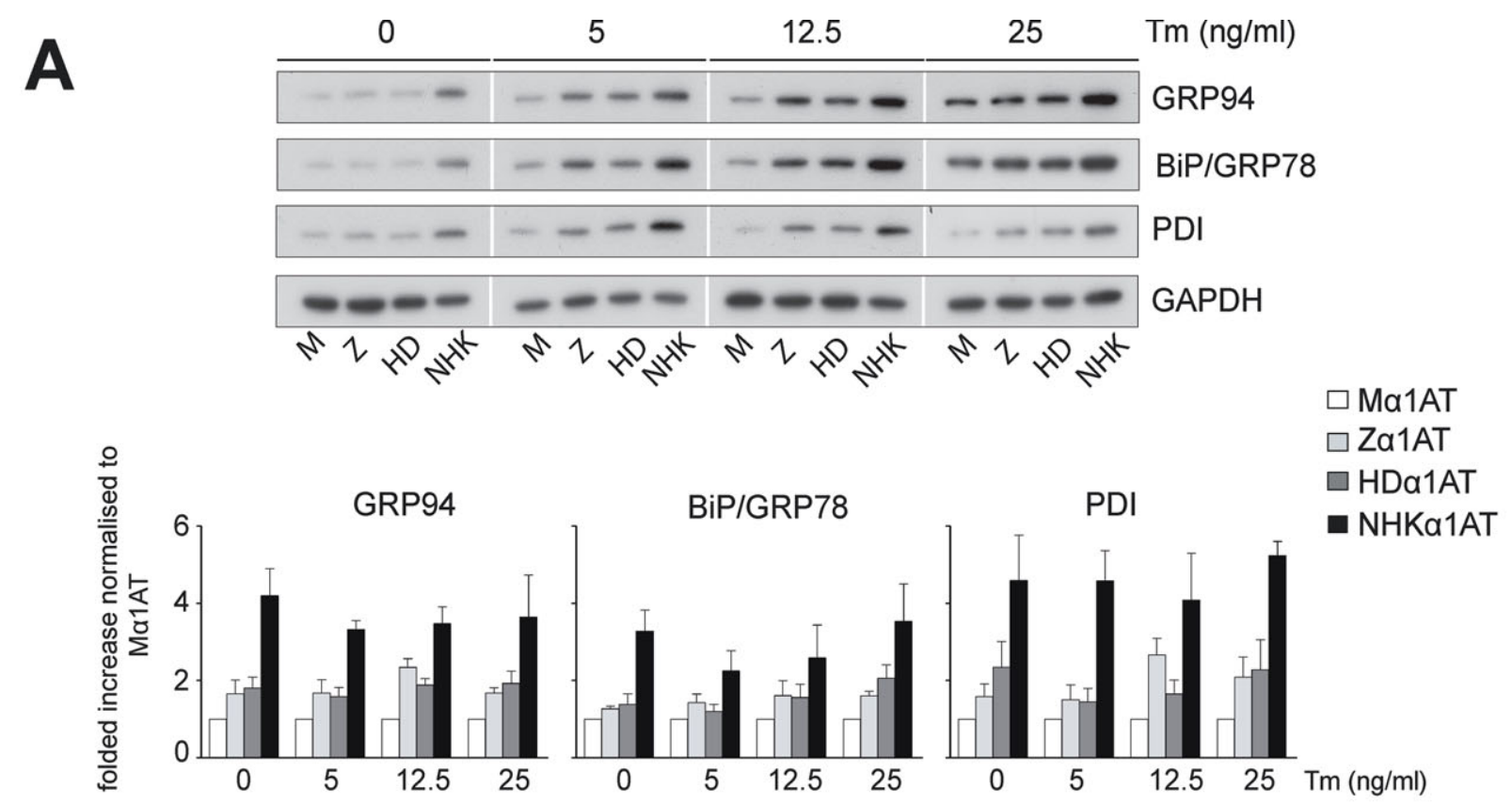

B
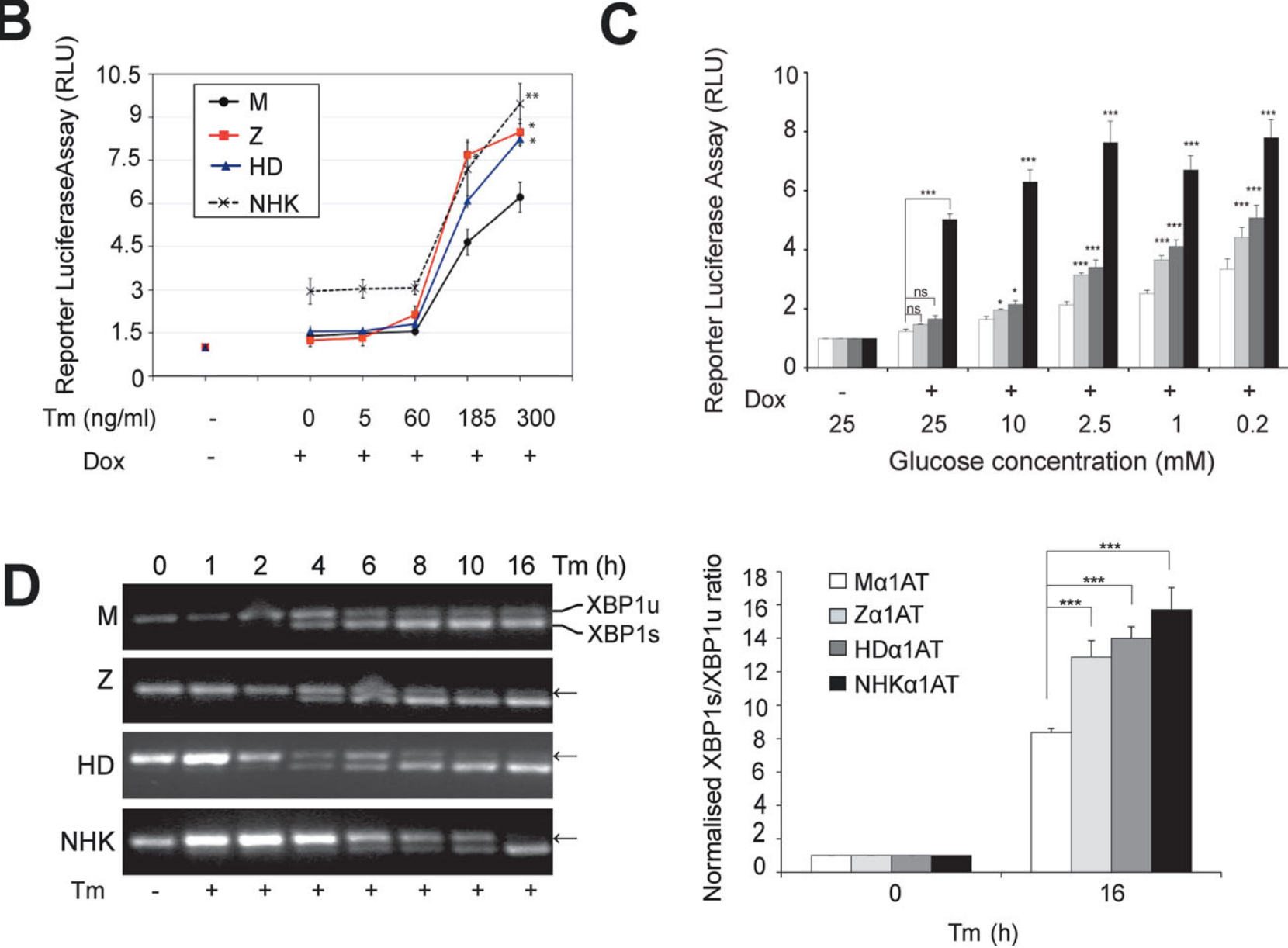

Fig. 5. Differential activation of three UPR sensors in polymeric forms of $\alpha 1$ AT reveals an increased sensitivity to UPR after a second hit. (A) Immunoblotting of whole cells lysates from CHO-K1 Tet-On cells induced with Dox for 48 hours and treated with the indicated doses of Tm for 16 hours. Grouping of separated lanes within the same gel was performed and indicated by dividing white lines. Histogram representation of four independent replicates of experiment (A) showing fold increase of GRP94, BiP/GRP78, or PDI normalized to loading control and then to WT variant for each concentration of tunicamycin (mean \pm standard error of the mean; SEM). (B) and (C) ATF6 activation during induction of ER stress. $\alpha 1$ AT expression was induced with Dox for 48 hours. Twenty-four hours before lysis, cells were cotransfected for 6 hours with a p5 $\times$ ATF6Luc encoding firefly luciferase under the control of a UPR response element and with pRL-TK Renilla luciferase as a normalization control, then treated with the indicated doses of Tm (B) or glucose $(C)$ for 16 hours. Graphs show firefly luciferase normalised to Renilla luciferase as mean \pm SEM $(n=3)$. (D) XBP1 processing during ER stress induction. Cells were induced with Dox for 48 hours and subjected to a time course of Tm treatment ( $300 \mathrm{ng} / \mathrm{mL}$ ). RT-PCR was used to amplify XBP1 mRNA, and PCR products were resolved by $2 \%$ (w/v) agarose gel electrophoresis. Ratio of spliced/unspliced XBP1 mRNA for each $\alpha 1$ AT variant after 16 -hour Tm is represented graphically as mean \pm SEM $(n=3)$. $* P<0.05$; ${ }^{* *} P<0.01 ; * * * P<0.001$, according to analysis of variance test, followed by Bonferroni's post-hoc test. 
Tubular ER
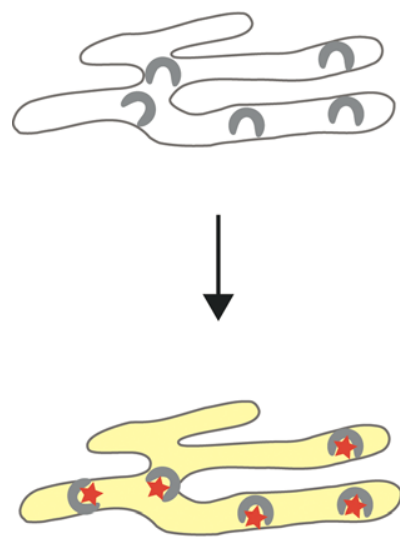

second insult
Vesiculated ER
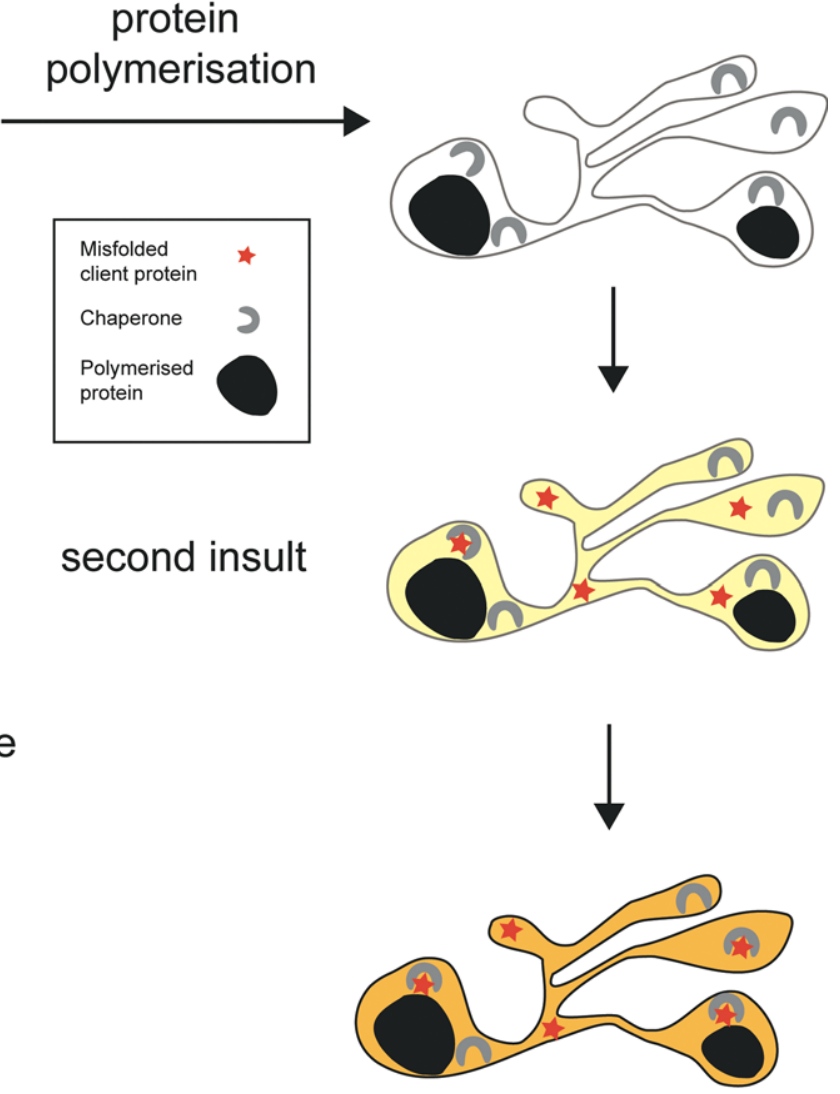

unresolved ER stress
Fig. 6. Model for enhanced sensitivity to ER stress observed in cells experiencing ER overload. An insult resulting in misfolded client protein is effectively buffered by chaperone diffusion in healthy cells. This prevents ER stress ("resolved"). In contrast, in cells that accumulate polymerized protein, the global ER environment is affected, which impairs chaperone access to misfolded proteins, thereby increasing the cell's propensity to experience ER stress ("unresolved"). Yellow represents a resolved ER stress, whereas orange corresponds to an unresolved ER response to ER overload. stress resolved
mRNA persisted after 16-hour treatment with $\mathrm{Tm}$ in cells expressing both $\mathrm{Z}$ and H334D $\alpha 1 \mathrm{AT}$, compared to the WT protein.

It is possible that differences in sensitivity to $\mathrm{Tm}$ might be responsible for the enhanced UPR activation in cells that express polymers of $\alpha 1 \mathrm{AT}$. However, this was not the case because treatment with Tm resulted in equal levels of a slower migrating glycosylated form of $\alpha 1 \mathrm{AT}$ and a faster migrating nonglycosylated form in each $\alpha 1$ AT-expressing cell line (Supporting Fig. 4).

\section{Discussion}

It is well recognized that point mutations of $\alpha 1 \mathrm{AT}$ form ordered polymers that are retained as inclusions within the ER, resulting in ER dysfunction and liver disease. ${ }^{8}$ These inclusions form in the face of effective disposal of misfolded monomer and polymers by ERAD $^{18,22}$ and autophagy. ${ }^{23}$ One of the cellular consequences of the accumulation of these ordered polymers is ER overload response (EOR), which results in the release of ER calcium and the activation of nuclear factor kappa B, a central mediator of inflamma- tion. ${ }^{19,24}$ This pathway is distinct from the canonical UPR, which is triggered by the accumulation of misfolded protein and results in the inhibition of protein synthesis, induction of ER chaperones, and ER stress. ${ }^{25}$ EOR and UPR pathways often occur together, but mutants of $\alpha 1 \mathrm{AT}$ provide ideal tools to probe these pathways because the polymer-forming mutants activate only the EOR, ${ }^{16-19}$ whereas truncated mutants activate only the UPR. ${ }^{7}$

We have used two polymeric mutants of $\alpha 1 \mathrm{AT}$ (E342K and H334D) to determine the mechanism by which ER overload can affect organization of the ER environment and the cell's susceptibility to ER stress. Our data reveal that polymers of $\alpha 1 \mathrm{AT}$ accumulate in spherical, apparently vesiculated ER, concomitantly with loss of the normal branching tubule network. Similar ER vesiculation has been reported to be associated with other cellular stresses, including mechanical injury and elevated cytosolic calcium concentration, although the physiological relevance of this remains unclear. $^{26,27}$ Live-cell imaging revealed that accumulation of polymers in vesiculated ER leads to a marked, specific impairment of luminal protein mobility 
resulting from an increased ER heterogeneity, which might reflect a change in either luminal viscosity and/ or in tortuosity for ER-RFP diffusion within and between spherical inclusions. Interestingly, the truncated NHK mutant that causes classical ER stress (UPR) and is efficiently degraded by the proteasome showed a different ultrastructural change characterized by gross expansion of ER cisternae. This had no effect on luminal protein mobility. Current technology limits the area of photobleaching to slightly larger than the size of an inclusion. However, because effects of photobleaching were examined by FLIP throughout the cell, we are confident in these findings. It will be valuable, when the technology has improved sufficiently, to revisit the diffusion between individual inclusions.

In agreement with previous studies, ${ }^{16-19}$ we report that cells expressing polymers of either $Z$ or $\mathrm{H} 334 \mathrm{D}$ $\alpha 1$ AT do not show constitutive activation of the UPR. However, these cells do exhibit a hypersensitivity to both pharmacological and physiological ER stressors. To date, most studies that have attempted to detect ER stress in cells expressing mutant $\alpha 1$ AT have made use of methods such as the up-regulation of ER chaperones. Although providing important insights, such approaches have been unable to address how ER overload with polymers of $\alpha 1$ AT might affect molecular organization of the ER organelle. Our observation that enhanced sensitivity to ER stress after expression of polymerogenic $\alpha 1 \mathrm{AT}$ mutants correlates with the presence of marked changes in the biophysical features of the ER suggests a mechanism for this phenomenon (Fig. 6). Chaperones can readily diffuse to sites of protein misfolding in cells possessing a reticular, highly interconnected ER. In contrast, in cells experiencing ER overload, a comparable level of misfolded client protein cannot diffuse freely, decreasing their accessibly to quality-control proteins required for folding and transport. Therefore, we propose a model in which decreased mobility or availability of ER chaperones resulting from changes in diffusive features and/or obstruction caused by protein overload sensitizes the cell to subsequent activation of the UPR by a second hit that further increases the unfolded secretory protein burden. Our findings are directly relevant to the liver disease associated with polymerogenic mutants of a1AT. However, although polymer accumulation involves a gain of function associated with liver dysfunction, epidemiological studies have shown that only $10 \%$ of patients with $\alpha 1$ AT deficiency develop clinically apparent liver disease. ${ }^{28}$ It appears that additional, incompletely understood genetic and environmental factors contribute to this heterogeneity in hepatic phenotype. ${ }^{29,30}$ Our results suggest that polymer accumulation increases the vulnerability of the cell to a "second hit" that ultimately initiates liver disease in individuals with $\alpha 1 \mathrm{AT}$ deficiency. Moreover, the mechanism proposed is likely to be relevant to other serpinopathies, such as the neuronal toxicity associated with polymers of neuroserpin in the dementia, familial encephalopathy with neuroserpin inclusion bodies. ${ }^{31}$

Acknowledgment: The authors thank Dr. James Irving for his help in preparing the figures and movies. The authors thank Marianna Mela, Department of Medicine, University of Cambridge, for providing the human liver tissue.

\section{References}

1. Lomas DA, Carrell RW. Serpinopathies and the conformational dementias. Nat Rev Genet 2002;3:759-768.

2. Wu Y, Whitman I, Molmenti E, Moore K, Hippenmeyer P, Perlmutter DH. A lag in intracellular degradation of mutant alpha 1-antitrypsin correlates with the liver disease phenotype in homozygous PiZZ alpha 1-antitrypsin deficiency. Proc Natl Acad Sci U S A 1994;91: 9014-9018.

3. Lomas DA, Evans DL, Finch JT, Carrell RW. The mechanism of Z alpha 1-antitrypsin accumulation in the liver. Nature 1992;357: 605-607.

4. Miranda E, Pérez J, Ekeowa UI, Hadzic N, Kalsheker N, Gooptu B, et al. A novel monoclonal antibody to characterize pathogenic polymers in liver disease associated with alphal-antitrypsin deficiency. HepatoLOGY 2010;52:1078-1088.

5. Lomas DA, Finch JT, Seyama K, Nukiwa T, Carrell RW. Alpha 1antitrypsin Siiyama (Ser53-->Phe). Further evidence for intracellular loop-sheet polymerization. J Biol Chem 1993;268:15333-15335.

6. Lomas DA, Elliott PR, Sidhar SK, Foreman RC, Finch JT, Cox DW, et al. alpha 1-Antitrypsin Mmalton (Phe52-deleted) forms loop-sheet polymers in vivo. Evidence for the $\mathrm{C}$ sheet mechanism of polymerization. J Biol Chem 1995;270:16864-16870.

7. Sifers RN, Brashears-Macatee S, Kidd VJ, Muensch H, Woo SL. A frameshift mutation results in a truncated alpha 1-antitrypsin that is retained within the rough endoplasmic reticulum. J Biol Chem 1988; 263:7330-7335

8. Gooptu B, Lomas DA. Polymers and inflammation: disease mechanisms of the serpinopathies. J Exp Med 2008;205:1529-1534.

9. Marciniak SJ, Ron D. Endoplasmic reticulum stress signaling in disease. Physiol Rev 2006;86:1133-1149.

10. Irving JA, Ekeowa UI, Belorgey D, Haq I, Gooptu B, Miranda E, et al. The serpinopathies studying serpin polymerization in vivo. Methods Enzymol 2011;501:421-466.

11. Snapp EL, Sharma A, Lippincott-Schwartz J, Hegde RS. Monitoring chaperone engagement of substrates in the endoplasmic reticulum of live cells. Proc Natl Acad Sci U S A 2006;103:6536-6541.

12. Costantini LM, Fossati M, Francolini M, Snapp EL. Assessing the tendency of fluorescent proteins to oligomerize under physiologic conditions. Traffic 2012;13:643-649.

13. Snapp EL. Fluorescent proteins: a cell biologist's user guide. Trends Cell Biol 2009;19:649-655.

14. Snapp EL, Altan N, Lippincott-Schwartz J. Measuring protein mobility by photobleaching GFP chimeras in living cells. Curr Protoc Cell Biol 2003; Chapter 21:Unit 21.1.

15. Lippincott-Schwartz J, Snapp E, Kenworthy A. Studying protein dynamics in living cells. Nat Rev Mol Cell Biol 2001;2:444-456. 
16. Graham KS, Le A, Sifers RN. Accumulation of the insoluble PiZ variant of human alpha 1-antitrypsin within the hepatic endoplasmic reticulum does not elevate the steady-state level of grp78/BiP. J Biol Chem 1990;265:20463-20468.

17. Hidvegi T, Schmidt BZ, Hale P, Perlmutter DH. Accumulation of mutant alpha1-antitrypsin $\mathrm{Z}$ in the endoplasmic reticulum activates caspases- 4 and -12, NFkappaB, and BAP31 but not the unfolded protein response. J Biol Chem 2005;280:39002-39015.

18. Kroeger H, Miranda E, MacLeod I, Pérez J, Crowther DC, Marciniak SJ, Lomas DA. Endoplasmic reticulum-associated degradation (ERAD) and autophagy cooperate to degrade polymerogenic mutant serpins. J Biol Chem 2009;284:22793-22802.

19. Davies MJ, Miranda E, Roussel BD, Kaufman RJ, Marciniak SJ, Lomas DA. Neuroserpin polymers activate NF-kappaB by a calcium signaling pathway that is independent of the unfolded protein response. J Biol Chem 2009;284:18202-18209.

20. Carroll TP, Greene CM, O'Connor CA, Nolan AM, O'Neill SJ, McElvaney NG. Evidence for unfolded protein response activation in monocytes from individuals with alpha-1 antitrypsin deficiency. J Immunol 2010;184:4538-4546.

21. Marciniak SJ, Yun CY, Oyadomari S, Novoa I, Zhang Y, Jungreis R, et al. CHOP induces death by promoting protein synthesis and oxidation in the stressed endoplasmic reticulum. Genes Dev 2004;18: 3066-3077.

22. Teckman JH, Burrows J, Hidvegi T, Schmidt B, Hale PD, Perlmutter $\mathrm{DH}$. The proteasome participates in degradation of mutant alpha 1-antitrypsin $\mathrm{Z}$ in the endoplasmic reticulum of hepatoma-derived hepatocytes. J Biol Chem 2001;276:44865-44872.
23. Hidvegi T, Ewing M, Hale P, Dippold C, Beckett C, Kemp C, et al. An autophagy-enhancing drug promotes degradation of mutant alpha1-antitrypsin Z and reduces hepatic fibrosis. Science 2010;329: 229-232.

24. Pahl HL, Baeuerle PA. The ER-overload response: activation of NF-kappa B. Trends Biochem Sci 1997;22:63-67.

25. Kaufman RJ. Orchestrating the unfolded protein response in health and disease. J Clin Invest 2002;110:1389-1398

26. Raeymaekers L, Lariviere E. Vesicularization of the endoplasmic reticulum is a fast response to plasma membrane injury. Biochem Biophys Res Commun 2011;414:246-251.

27. Subramanian K, Meyer T. Calcium-induced restructuring of nuclear envelope and endoplasmic reticulum calcium stores. Cell 1997;89: 963-971.

28. Sveger T. The natural history of liver disease in alpha1-antitrypsin deficient children. Acta Paediatr Scand 1995;77:847-851.

29. Pan S, Huang L, McPherson J, Muzny D, Rouhani F, Brantly M, et al. Single nucleotide polymorphism-mediated translational suppression of endoplasmic reticulum mannosidase I modifies the onset of end-stage liver disease in alpha1-antitrypsin deficiency. Hepatology 2009;50: 275-281.

30. Perlmutter DH. Liver injury in $\alpha 1$-antitrypsin deficiency: an aggregated protein induces mitochondrial injury. J Clin Invest 2002;110: 579-1583.

31. Davis RL, Shrimpton AE, Holohan PD, Bradshaw C, Feiglin D, Collins $\mathrm{GH}$, et al. Familial dementia caused by polymerization of mutant neuroserpin. Nature 1999;401:376-379. 